\title{
Molecular Simulations for Anti-amyloidogenic Effect of Flavonoid Myricetin Exerted against Alzheimer's $\beta$-Amyloid Fibrils Formation
}

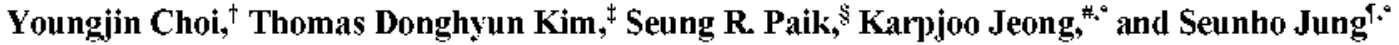 \\ ${ }^{\dagger}$ Biochip Research Center, Hoseo Linwersity, Asan 336-795, Korea \\ ${ }^{\ddagger}$ Department of Biochemistry Liniversity of Chicago, Chicago, Ihinois 60637, U.S.A \\ \$School of Chemical and Biological Engineering, College of Engineering, Seoul National Lniversitw, Seoul 151-74t, Korea \\ "College of Information and Communication \& Department of Adwanced Technologv Fusion, Konkuk Lniversity, \\ Seoul 143-701, Korea. ${ }^{*}$ E-mail: jeongkakonkuk.ac.kr \\ "Bion folecutar Informatics Center \& Department of Bioscience and Biotechnologv, Konkuk Lhiversity, Seoul 143-701, Korea \\ ${ }^{\star} E$-mail: shitungakonkuk:ackr \\ Received June 27, 2008
}

\begin{abstract}
Comparative molecular simulations were performed to establish molecular interaction and inhibitory effect of flavonoid myricetin on formation of amyloid fibris. For computational comparison. the conformational stability of myricetin with amyloid $\beta$-peptide (A $\beta$ ) and $\beta$-amyloid fibrils (fA $\beta$ ) were traced with multiple molecular dy namics simulations (MD) using the CHARMM program from Monte Carlo docked structures. Simulations showed that the inhibition by myrricetin involves binding of the flavonoid to $\mathrm{f} A \beta$ rather than $\mathrm{A} \beta$. Even in $\mathrm{MD}$ simulations over $5 \mathrm{~ns}$ at $300 \mathrm{~K}$. myricetin/fA $\beta$ complex remained stable in compact conformation for multiple trajectories. In contrast. myricetin/A $\beta$ complex mostly tumed into the dissociated conformation during the $\mathrm{MD}$ simulations at $300 \mathrm{~K}$. These multiple $\mathrm{MD}$ simulations provide a theoretical basis for the higher inhibitory effect of myrricetin on fibrillogenesis of $\mathrm{f} A \beta$ relative to $\mathrm{A} \beta$. Significant binding between myricetin and $\mathrm{fA} \beta$ observed from the computational simulations clearly reflects the previous experimental results in which only fA $\beta$ had bound to the my ricetin molecules.
\end{abstract}

Key Words : Amy loid fibril, Conformational analysis. Flavonoid, Molecular dynamics simulations, Myricetin

\section{Introduction}

Amyloidosis is a clinical condition marked by the deposition of fibrous amyloids in various organs and tissues of the body. ${ }^{3}$ The protein fibril formation may be causative for various fatal human disorders including Alzheimer's disease $(\mathrm{AD})^{2}$ Common structural feature of amyloid fibrils has been characterized by critical cross $\beta$-sheet core structure that perpetuates along the fibril long axis. ${ }^{3}$ Fibrillogenesis of the monomeric anyloid $\beta$-peptide $(\mathrm{A} \beta$ ) found in $\mathrm{AD}$ generally occurs via nucleation-dependent polymerization. ${ }^{+}$ If supersaturated $A \beta$ solution forms a nucleus, the growth of fibril is accelerated to evolve into the $\beta$-anyloid fibrils $(\mathrm{fA} \beta) .{ }^{5}$ The structure-toxicity relationship study performed by Riek and co-workers indicates that $\mathrm{fA} \dot{\beta}$ contain an intrinsic toxicity that correlates with their morphologies as well as quantities. ${ }^{6}$ They showed that the neurotoxicity was generally proportional to the size of $\mathrm{fA} \beta$. Therefore. it would be crucial to regulate the fA $\beta$ growth by chemical additives to develop potent anti-Alzheimer's disease drugs.

Recent studies for the $\mathrm{fA} \beta$ fibrilization revealed that some flavonoid compounds are able to inhibit the fibril growth and destabilize the preformed fA $\beta{ }^{7}$ Ono et $a$ l. found that myricetin exhibited its anti-amyloidogenic effect via preferential binding to the $\mathrm{fA} \beta$ rather than the $\mathrm{A} \beta$ monomers. In order to develop anti-amyloidogenic drugs. it is mandatory for structural biologists to acquire a detailed understanding of molecular interactions between flavonoids and
fA $\beta$.

In this report comparative molecular simulations were performed for both myricetin/A $\beta$ and myricetin/ $\mathrm{AA} \beta$ in order to explain the experimentally observed inhibitory effect of myricetin for the fibril growth of $A \beta$. To reproduce real interactions between myricetin and anyloid peptide. Monte Carlo (MC) docking and molecular dynamics (MD) simulations were carried out for the molecular models of myricetin $/ \mathrm{A} \beta$ and myricetin/fA $\beta$. Significant binding between myricetin and $\mathrm{f} A \beta$ observed from the computational simulations clearly reflects the previous experimental results ${ }^{8}$ in which only $\mathrm{fA} \beta$ bind to the myricetin molecules. From the simulation of fibrillar $\mathrm{fA} \beta$ with myricetin. it has been found that the myricetin interactions with the outermost part of fibrils are responsible for the experimentally observed inhibitory effect on the fibrillogenesis of fA $\beta$.

\section{Results and Discussion}

Differences in docked conformations and energetics of the myricetin with $\mathbf{A} \beta$ and $\mathbf{f} A \beta$. The interaction energy profiles of each myricetin complex with $A \beta$ and $\mathrm{f} A \beta$ during MC docking simulations are shown in Figure 1. The intermolecular energy between myricetin and $\mathrm{A} \beta$ was -18.83 $\mathrm{kcal} / \mathrm{mol}$ and those of myricetin and $\mathrm{fA} \beta$ was $-21.41 \mathrm{kcal} /$ mol during last $6 \times 10^{5} \mathrm{MC}$ trials. This means that the myricetin molecule forms a more stable complex with fA $\beta$ than the monomeric $\mathrm{A} \beta$. This result seems to be consistent 


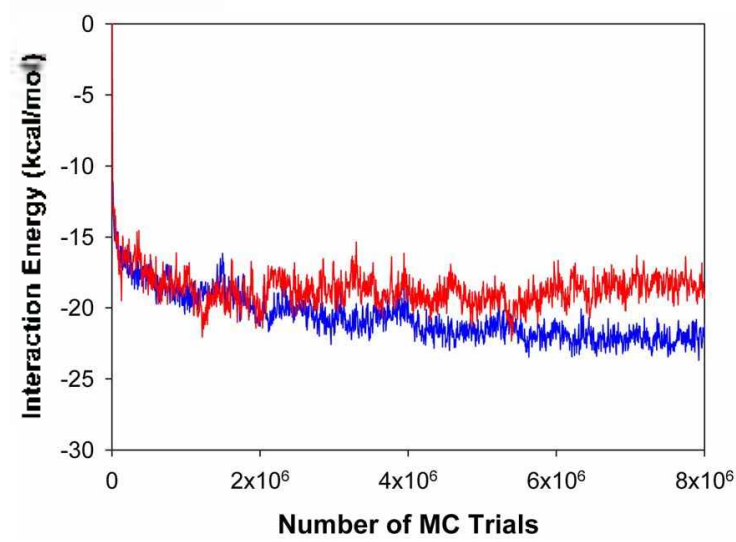

Figure 1. Interaction energy profiles of myncetin with helical (red line) or fibrous (blue line) form of amyloid peptide during $\mathrm{MC}$ docking simulations.

with the experimental observation that the flavonoid myricetin exerted its binding ability only to $\mathrm{f} \beta$ rather than $A \beta$. Interaction energy profiles of the myricetin/A $\beta$ and the myricetin/fA $\beta$ are shown in Figure 2. Myricetin was docked onto a region between $\alpha$-helix and $C$-termini of the $A \beta$. There was no special binding site on the $A \beta$ and the myricetin just clung to the surface of the peptide (Fig. 2). Contrary to the case of nyricetin/A $\beta$, the myricetin formed a stable docked complex with the fA $\beta$. The $U$-shaped conformation of $\mathrm{fA} \beta$ provided suitable binding site for a flat shaped myricetin molecule. ${ }^{5}$ The myricetin was fully stuck into the hydrophobic binding patch of the $\mathrm{fA} \beta$. These two conformations were further investigated by MD simulations.

(a)

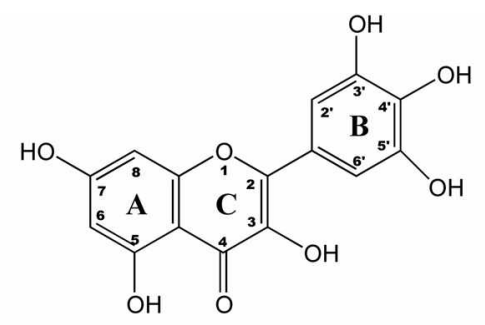

(b)

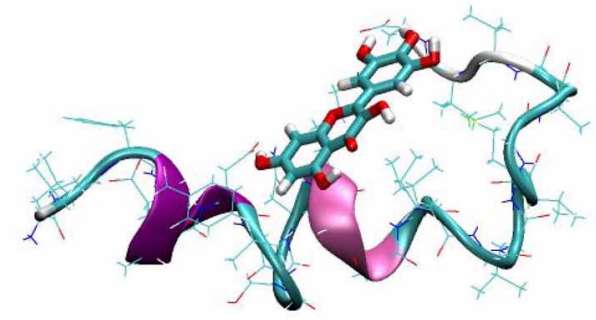

(c)

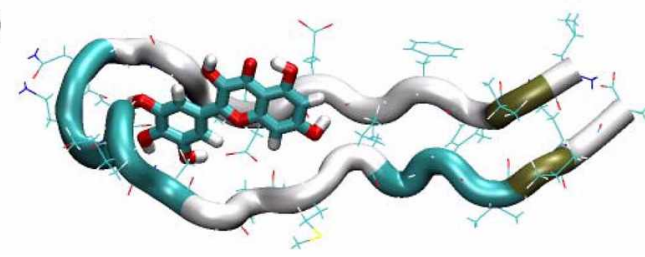

Figure 2. The 2-D structure of myricetin (a) and its docked conformation with helical (b) or fibrous (c) form of amyloid peptide.

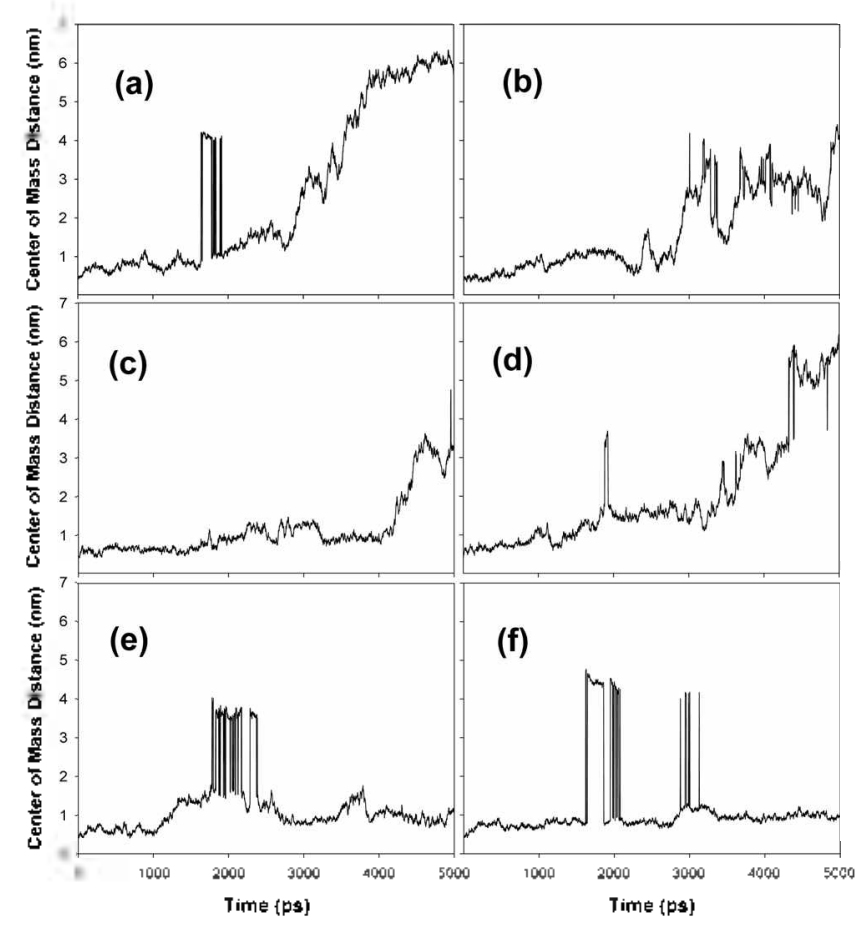

Figure 3. Center of mass distances between myricetin and helical tom of amy loid during multiple 5ns-MD simulations.

Complex stability of myricetin/A $\beta$ and myricetin/fA $\beta$ during multiple $\mathrm{MD}$ simulations. Multiple $\mathrm{MD}$ simulations were perfonmed from the lowest energy conformation of $\mathrm{MC}$ simulations. These MD simulations for each myricetin complex evidenced more stable conformation of myricetin/ $/ \mathrm{A} \beta$ compared to myricetin/A $\beta$. Figure 3 shows center of mass distance between myricetin and $\mathrm{A} \beta$ during 5-ns of $\mathrm{MD}$ simulations for six trajectories. Four trajectories showed gradual dissociation of the myricetin from $\mathrm{A} \beta$ with some variations (Figure 3a-d). Stable docked confonmations were only maintained for just two trajectories with showing highfluctuations (Figure 3e f). Therefore, we can conclude that the interaction between myricetin and $\mathrm{A} \beta$ may be weak and unsuitable for keeping the complex stability during $M D$ simulations. Compared with the myricetin $/ \mathrm{A} \dot{\beta}$ complex. the myricetin showed a more stable structure with the $\mathrm{fA} \beta$ during multiple MD simulations. Final dissociation of myricetin from $\mathrm{fA} \beta$ were observed for only two trajectories (Figure 4c. d). Others were tightly associated with each other during MD simulations. in which averaged center of mass distances were $0.83 .0 .70,0.53$, and $0.52 \mathrm{~mm}$. respectively (Figure 4a, b. e. f).

The differences in the confonmational stability of the myricetin/ $\beta$ and the myricetin/fA $\beta$ were also supported by minimum distances between the myricetin and $\mathrm{A} \beta$ or $\mathrm{fA} \beta$ (Figure 5). After 5-11s of MD simulations. trajectory-averaged minimum distance between myricetin and $A \beta$ was about $3 \mathrm{~nm}$, while the distance between myricetin and $\mathrm{fA} \beta$ was below $2 \mathrm{~nm}$. This means that the atomic interactions between myricetin and $\mathrm{fA} \beta$ were more stable than those of myricetin and $\mathrm{A} \beta$. Figure 6 is a solvent-accessible surface area change upon formation of complexes between myri- 


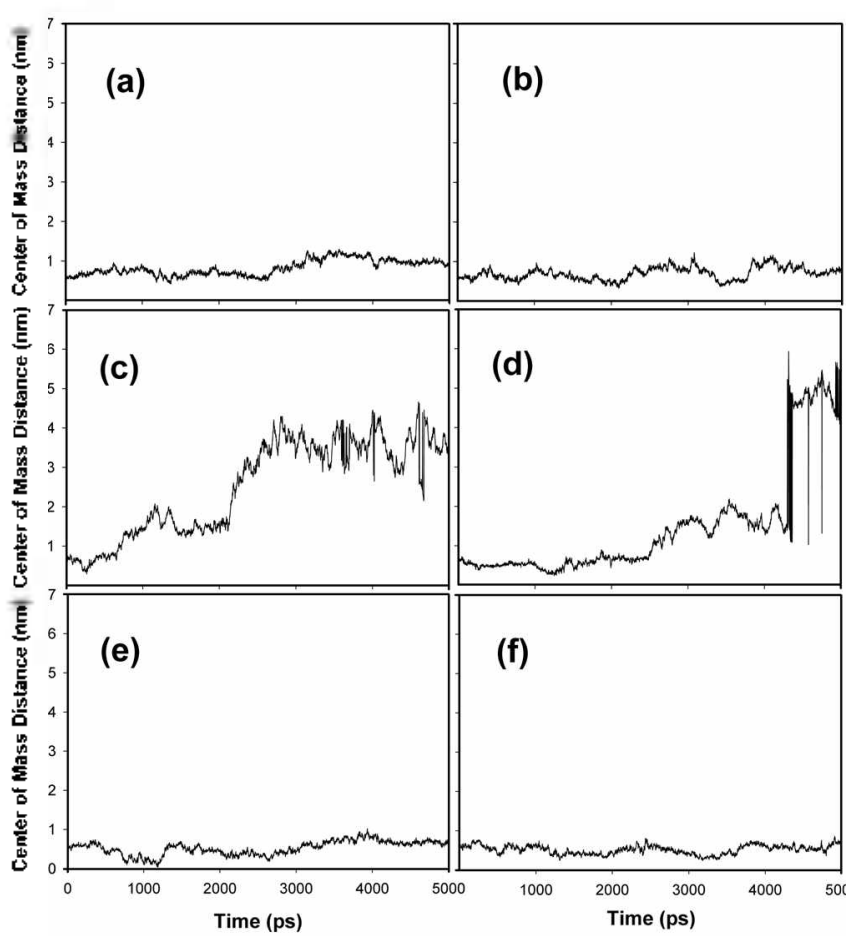

Figure 4. Center of mass distances between myncetin and fibrous form of amvloid during multiple 5ns-MD simulations.

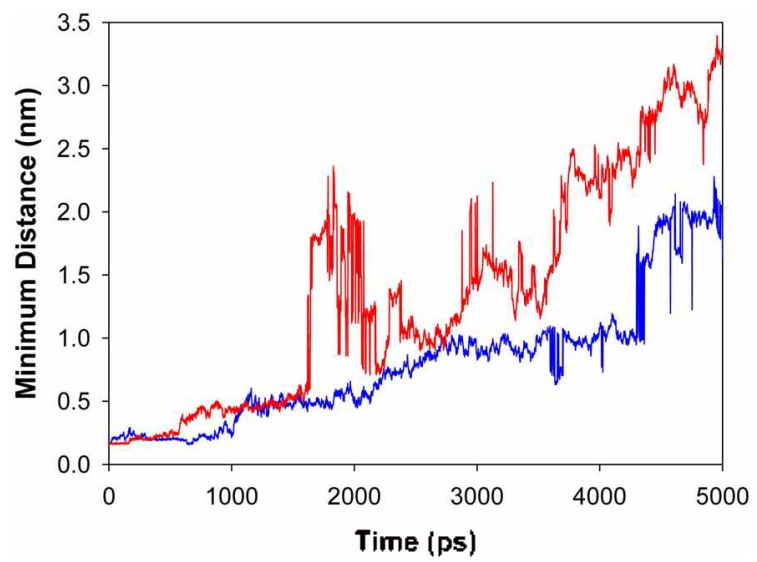

Figure 5. Averaged mininum distance between myricetin and helical (red line) or fibrous (blue line) form of amyloid peptide during multiple MD simulations.

cetin and $\mathrm{A} \beta$ or $\mathrm{fA} \beta$. At early $\mathrm{MD}$ phase $(0-1 \mathrm{~ns})$, the surface area change from binding of myricetin to fA $\beta$ was $-5.85 \mathrm{~nm}^{2}$. This indicates that the surface area was decreased by approximately $5.85 \mathrm{~nm}^{2}$ due to the binding of inyricetin and $\mathrm{fA} \beta$. The value was increased to $-3.70 \mathrm{~nm}^{2}$ during late MD simulation phase ( $4-5 \mathrm{~ns})$. For the myricetin/ $A \beta$. the surface area change was $-4.40 \mathrm{~cm}^{2}$ during early MD phase and the value was increased to $-1.33 \mathrm{~nm}^{2}$ during late MD phase. The surface area change of myricetin/ $\mathrm{fA} \beta$ was much lower than that of myricetin/A $\beta$ at any MD time phase. These results suggest tighter binding of myricetin to the $\mathrm{f} A$ in comparison to $A \beta$. The ratio of surface area changes during late to early $\mathrm{MD}$ phase was 0.63 for the myricetin/fA $\beta$ and 0.30 for the myricetin/A $\beta$. This means

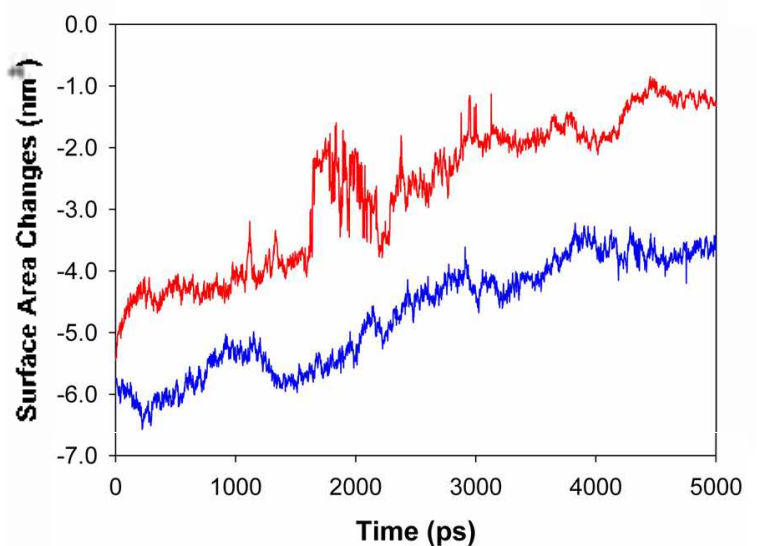

Figure 6. Averaged surface area change upon complexation of myricetin with helical (red line) or fibrous (blue line) form of amyloid peptide during multiple MD simulations.

that the surface area decrease of myricetin $/ \mathrm{A} A \beta$ was two-fold higher than that of myricetin $/ \mathrm{A} \beta$ during the time scale of $\mathrm{MD}$ simulations. Therefore, binding stability of myricetin to the $\mathrm{fA} \beta$ is more tolerant to dynamic fluctuation of the molecular complex during the MD simulation time. The strong binding ability of myricetin to $\mathrm{A} A \beta$ in comparison to $\mathrm{A} \beta$ could provide a molecular understanding for experimentally observed inlibitory effect of myricetin on anyloid fibrilliogenesis. (a)
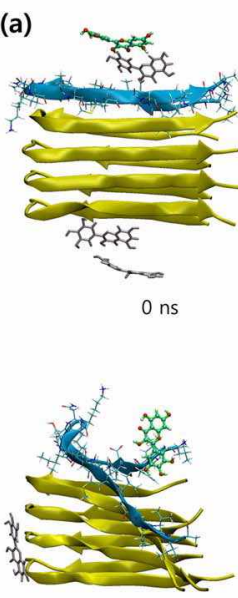

$1.85 \mathrm{~ns}$

(b)

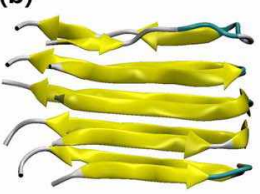

$0 \mathrm{~ns}$

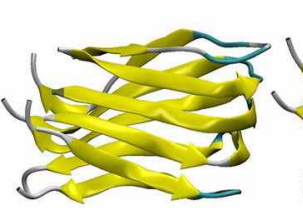

$3.2 \mathrm{~ns}$
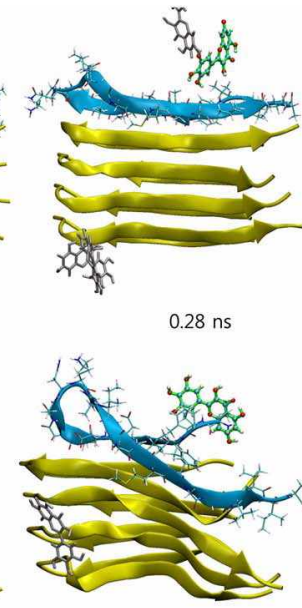

$2.69 \mathrm{~ns}$

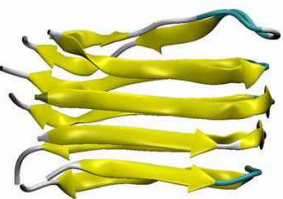

$0.28 \mathrm{~ns}$

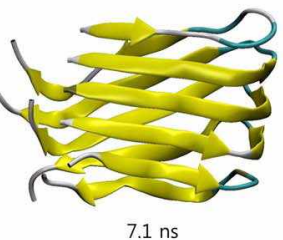

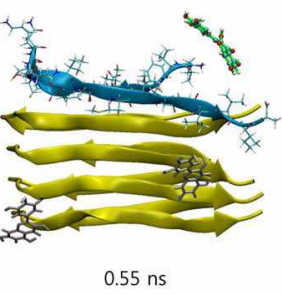

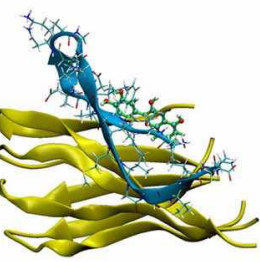

811 ns

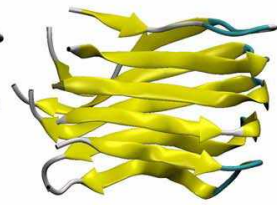

$0.55 \mathrm{~ns}$

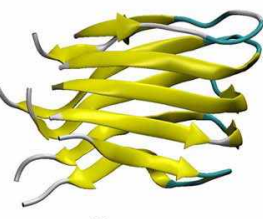

10 ns
Figure 7. Destabilization of $\mathrm{f} A \bar{\beta}$ fibrils with myricetin (a) and stable $\mathrm{fA} \beta$ tibrils (b) during $10 \mathrm{~ns} \mathrm{MD}$ simulations. 
Destabilization of $\mathrm{fA} \beta$ by myricetin during the MD simulations. Myricetin can destabilize existing fibrils as well as have inhibitory effect on formation of emerging fibrils. Figure 7 is the time course of the fibril destabilization by myricetin during 10-ns of MD simulations. The fibrils were modeled as five sets of $\mathrm{fA} \beta$ monomers. After $280 \mathrm{ps}$. the myricetin made an interaction with the outermost $\mathrm{fA} \beta$ sheaf. After $550 \mathrm{ps}$, the myricetin had bound to C-termini of the fA $\beta$ sheaf $v i a$ hydrophobic interaction of aromatic rings. The binding interaction was intensified into the core cavity of the outermost $\mathrm{fA} \beta$ sheaf after $1.85 \mathrm{~ns}$ of MD simulation. In that time, about half of the domain of $\mathrm{fA} \beta$ sheaf was segregated from remaining fA $\beta$ fibrils. The level of interaction between fibrils and $\mathrm{f} A \beta$ part was minimized by the bound myricetin. After $2.69 \mathrm{~ns}$, the myricetin moved out of $N$-termini of $\mathrm{fA} \beta$ sheaf and showed relatively decreased interaction with the fA $\beta$ sheaf. Finally. the nyricetin moved to the next part of $\mathrm{f} A \beta$ sheaf to destabilize remaining part of $\mathrm{fA} \beta$ fibrils after $8.11 \mathrm{~ns}$. From the MD simulation snapshots in the Figure 7. it is evidenced that the myricetin exert an anti-amyloidogenic effect by destabilizing the $\mathrm{f} A \beta$ fibrils as well as inhibiting the formation of fibrils from monomeric peptides

For the quantitative comparison between with and without myricetin conditions. $\mathrm{C} \alpha$ atonic distance was analyzed for each fA $\beta$ sheaf in the fibrils. Figure 8 is time course of interatomic distance between $\mathrm{f} A \beta$ sheaf $1-2,2-3,3-4$, and 4 5 for centric $\mathrm{C} \alpha$ atom of each Gly 13 residue. During the MD sumulations. it was found the interatomic distances of outer fA $\beta$ sheaf 1.2 or $4-5$ were dramatically increased by myricetin (Figure 8a. d). However the distances for the inner fA $\beta$ sheaf were not changed during the MD simulations for both with and without myricetin conditions (Figure 8b. c). It seems that myricetin molecule exert anti-amyloidogenic effect for outer part of $\mathrm{f} A \beta$ fibrils by perturbing these intershelf $\mathrm{vdw}$ interactions. Figure 9 is intermolecular electrostatic and vdw energy between outermost $f A \beta$ sheaf $I$ and 2 .

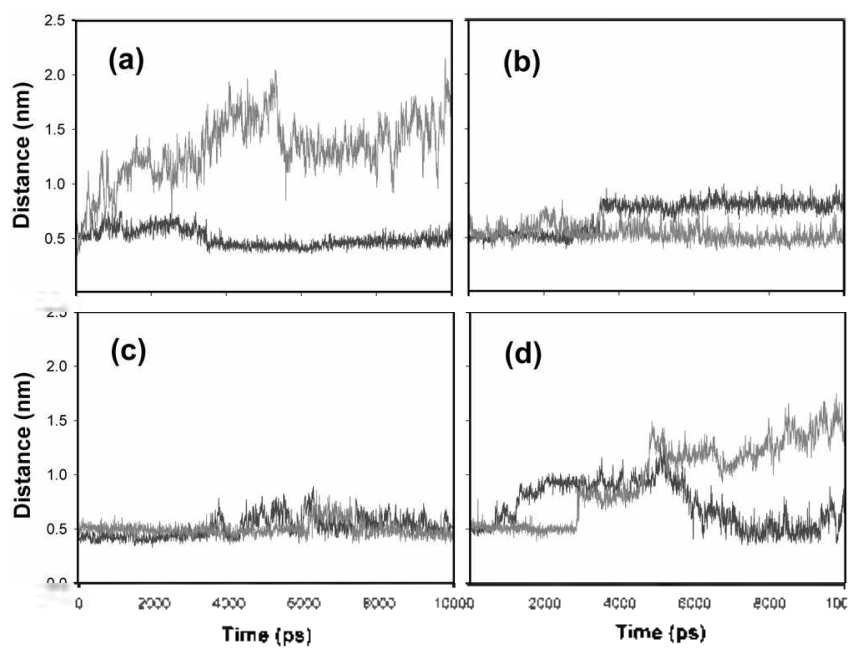

Figure 8. Interatomic $\mathrm{C} \alpha$ distances between $\mathrm{IA} \beta$ sheaf 1 and 2 (a), 2 and 3 (b), 3 and 4 (c), 4 and 5 . (d). Red line indicates $A A \beta$ tibrils with myricetin and blue line indicates $\mathrm{f} A \beta$ fibrils alone.

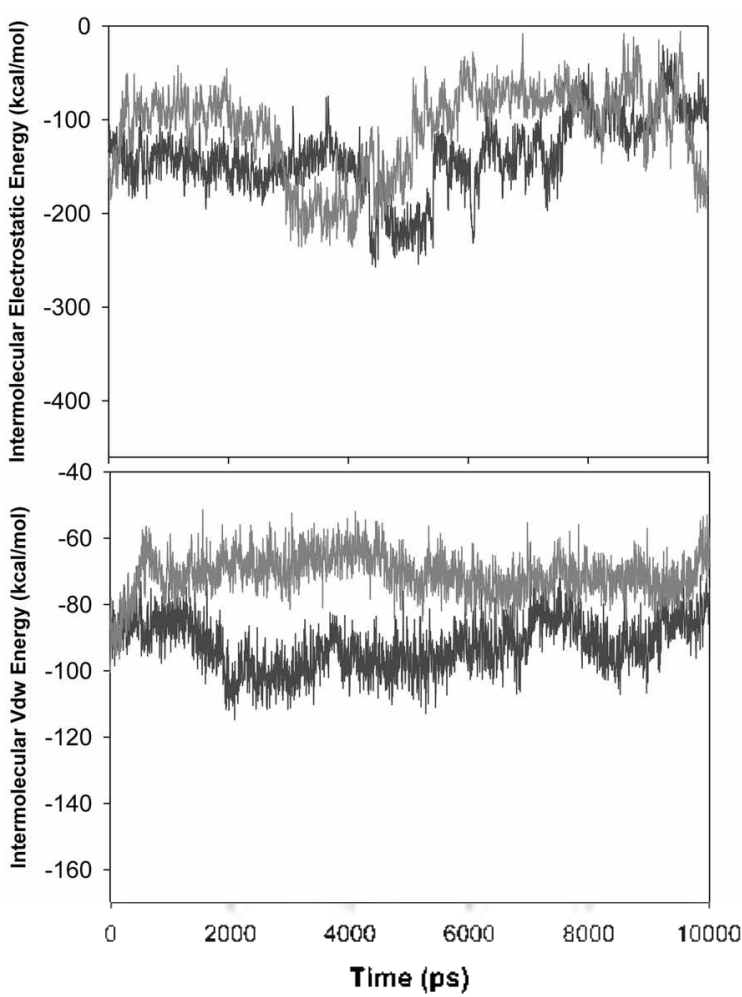

Figure 9. Intermolecular electrostatic (a) and vdw energy (b) between outenmost fA $\beta$ sheaf. Red line indicates $\mathrm{fA} \beta$ fibrils with myricetin and blue line indicates tA $\beta$ tibrils alone.

During MD simulations. the intermolecular electrostatic energies were controversial to each $\mathrm{fA} \beta$ fibril with and without myricetin (Figure 9a). However. intermolecular vdw energies were discriminated between the $\mathrm{fA} \beta$ fibrils (Figure $9 \mathrm{~b})$. The vdw energy of $\mathrm{fA} \beta$ fibrils was lowered than the case of $\mathrm{fA} \beta$ fibrils with myricetin system during the simulation times. Intermolecular vdw energy was $-91.2 \mathrm{kcal} / \mathrm{mol}$ for the $\mathrm{fA} \beta$ fibrils without myricetin and $-71.3 \mathrm{kcal} / \mathrm{mol}$ for the $\mathrm{fA} \beta$ fibrils with myricetin. respectively. That means myricetin molecule destabilize the intermolecular $\mathrm{vdw}$ interaction between outer part of $\mathrm{fA} \beta$ sheaf rather than interior part of $\mathrm{fA} \beta$ fibrils.

\section{Methods of Computation}

Construction of the molecular models and protocol of MC docking simulations. The starting configuration of each $\mathrm{A} \beta$ and $\mathrm{fA} \beta$ for $\mathrm{MC}$ simulations was obtained from the Protein Data Bank (PDB). The PDB ID's for $\mathrm{A} \beta$ and $\mathrm{AA} \beta$ were lzoq and 2 beg. respectively. To obtain representative conformations of the 3D coordinate ensemble, the Discovery Studio/Builder module (version 1.7. Accelrys Software Inc.) was used as a molecular editor. The atomic coordinates and atomic partial charges of the myricetin were obtained by utilizing $a b$ initio calculations with $6-311 \mathrm{G}$ basis set at Hartree-Fork (HF) level. All simulations were perfomed using a general molecular modeling program. CHARMM (version $30 \mathrm{bl}$ ). with a parm 22 all-atom force field. ${ }^{\mathrm{s}}$ The $\mathrm{MC}$ docking simulations were performed using the "MC" module 
of CHARMM. The short-range non-bonding interactions were truncated with a $13-\dot{A}$ cutoff. An implicit solvent water model was used with a distance-dependent dielectric constant. The docking process was assumed to be a $1: 1$ interaction between each anyloid peptide and myricetin during the $\mathrm{MC}$ runs. The initial configuration of each amyloid and myricetin molecule was positioned arbitrarily within a neighboring distance. Trials to a new configuration were accomplished by changing each move set of a guest molecule. The MC nove set for flexible docking was composed of rigid translations. rigid rotations. and rotations of freely rotatable dihedral angles of the both myricetin and amyloids. A single step consists of picking a random conformer. making a random move, minimizing the energy of a new conformer. and then checking the energy with a Metropolis criterion. ${ }^{16}$ The $\mathrm{MC}$-accepted structures were saved every 4000 steps for 8.000 .000 trials. Multiple 10-MC trajectories were generated with different random number seed to convergence of the simulation. These MC processes produced convergent docked structures for each monomeric anyloid with myricetin.

Multiple molecular dynamics simulations of the each myricetin/ $/ \mathbf{A}$ and myricetin/f $\mathbf{A} \beta$ complexes. The starting configurations of each myricetin/ $A \beta$ and myricetin/ $\mathrm{fA} \beta$ complex for the MD sinulations in explicit water were taken from the MC-docked conformations ${ }^{11}$ with the lowestenergy value (Figure 2). The geometries of these molecular models were fully optimized before MD runs. A TIP3P three-site rigid water model was used to solvate the complexes. ${ }^{12}$ Water molecules were removed if they were closer than $2.8 \AA$ to any heavy atoms of the complexes. In summary, each system was constructed using periodic boundary conditions with a cubic box of dimensions $50 \AA \times 50 \AA \times$ $50 \AA$. consisting of myricetin/ $A \beta$ complex and 4,320 water. or myricetin/fA $\beta$ complex and 4,293 water molecules. The system was minimized by 2.000 steps of conjugate gradient. followed by Adopted Basis Newton-Raphson until the rootmean-square gradient was less than $0.001 \mathrm{kcal} / \mathrm{mol}$. The MD simulations were performed using the CHARMM 30b1 program in the isothermal-isobaric ensemble at $300 \mathrm{~K}(P=1$ bar. $T=300 \mathrm{~K}$ ). The particle mesh Ewald summation method $^{13}$ was used to treat the long-range electrostatic interactions. ${ }^{14}$ The bond lengths of all molecules were constrained with the SHAKE algorithm. ${ }^{15}$ The time step was 2.0 fs. and the non-bonded pair list was updated every 25 steps. The short-range non-bonded interactions were truncated with a 13-A cutoff. The temperature and pressure of the system were regulated using the Langevin piston method in conjunction with Hoover's thermostat. ${ }^{16}$ The system was gradually heated to each targeted temperature for $50 \mathrm{ps}$ and equilibrated at this temperature. The production MD trajectory with one snapshot per 2 ps was collected for 5.000 ps. Total six-independent MD simulations were performed with different random number seed to cover wide conformational sampling. For the simulation of whole fibrils with myricetin. molecular model was constructed with the cubic box of dimensions $70 \AA \times 70 \AA \times 70 \AA$, consisting of four myricetins. five-fA $\beta$ fibril. and 10.216 water molecules. The MD simulations were carried out over the course of 10 -ns to observe destabilization of $\mathrm{fA} \beta$ fibril by myricetin.

\section{Conclusions}

Comparison of the dynamics of myricetin/A $\beta$ and myricetin/fA $\beta$ complex during molecular simulations demonstrate that myriceyin disturb upon Alzheimer's $\beta$-amyloid fibrillogenesis by preferential binding into the hydrophobic patch of $\mathrm{fA} \beta$. Multiple MD simulations of myricetin $/ \mathrm{A} \beta$ and myricetin $/ \mathrm{fA} \beta$ provided the important theoretical insight into the experimentally determined anti-amyloidogenic effect of flavonoid compound, suggesting that myricetin could bind to fibrous form of any loid peptides, thereby decreasing favorable interaction between monomeric $\beta$-sheets of amyloid fibrils. Based on the present study, we conclude that computational methodology can make great contribution toward anti-any loidogenic drug design using natural flavonoid compounds.

Acknowledgments. This study was supported by a grant of the $e$-Science Project of KISTI (Korea Institute of Science and Teclunology Information) in MOST (Ministry of Science and Technology) and partly supported by a grant of the Korea Research Foundation (KRF-2006-005-J03402).

\section{References}

1. Pepys. M. B. Amm. Rev: Hed. 2006. 57. 223

2. Selkoe, D. J. Nanue $\mathbf{2 0 0 3}+26.900$.

3. Sunde. M; Serpell. L. C. Bartlam. M. J. Hol. Biol. 1997, 273.729.

4. Harper. J. D.; Lansbury, P. T. Jr. Amm. Rev. Biochem. 1997, 66. 385.

5. Rochet. T. C.: Lansbury. P. T. Ir. Cum Opin. Stmct Biol. 2000.10. 60 .

6. Luhrs, T.: Ritter, C.: Adrian, M; Riek-Loher. D; Bohrmann. B; Dobeli, H.: Schubert, D.; Riek. R. Proc. Nat Acad Sai LS. 2005. 29,17342 .

7. Ono. K:: Yoshiike. Y: Takashima. A.: Hasegawa. K.: Naiki. H.: Yamada. M. I. Newrochem. 2003. 87. 172.

8. Hirohata. M: Hasegawa. K.: Yasuhara. S. T.: Ohhashi. Y: Ookoshi, T;: Ono, K; Yamada, M.; Naiki, H. Biochemistry 2007. 46.1888 .

9. Brooks, B. R.: Bruccoler, R. E; Olafson, B. D.: States, D. J. Swaminathan. S.: Karplus. M. J. Contput. Chent 1983. 4. 187.

10. Metropolis. N.: Rosenbluth. A. W.: Rosenbluth. M. N.: Teller. A. H.: Teller. E. J. Chem. Phys. 1953. 21. 1087.

11. Choi. Y.: Park. S.; Jeong, K.: Jung. S. Bull Rorean Chem Soc. 2007. 28,1811

12. Jorgensen. W. L. J. Chem. Plys. 1983. 79.926.

13. Darden. T.: York. D.: Pedersen. L. J. Chem. Phns. 1993. 98. 10089 .

14. Lee. S. H. Bull. Korem Chem. Soc. 2006. 27. 1154.

15. Ryckaert. J. P. Cicootti. G; Berendsen. H. J. C. J. Comput. Phys. 1977. 23,327

16. Feller. S. E.: Zhang. Y; Pastor, R. W.: Brooks. B. R. J. Chem. Phos 1995. 103.4613. 